\title{
A systematic review and meta-analysis of the data behind current recommendations for corticosteroids for non-HIV related PCP; Knowing when you are on shaky foundations
}

\author{
Patil Injean ${ }^{1}$, Samantha J. Eells ${ }^{2,3}$, Hoover Wu ${ }^{4}$, Imani McElroy ${ }^{4}$, Aric Gregson ${ }^{4}$ and James A. McKinnell ${ }^{4,5,6 *}$ \\ ${ }^{1}$ Western University of Health Sciences, Pomona, California, USA \\ ${ }^{2}$ Department of Epidemiology, UCLA Jonathan and Karin Fielding School of Public Health, University of California, Los Angeles, California, USA \\ ${ }^{3}$ Science 37, Inc., Los Angeles, California, USA \\ ${ }^{4}$ David Geffen School of Medicine, University of California, Los Angeles, California, USA \\ ${ }^{5}$ Division of Infectious Diseases, LA-Biomed Research Institute at Harbor-UCLA Medical Center, Los Angeles, California, USA \\ ${ }^{6}$ Torrance Memorial Medical Center, Torrance, California, USA
}

\begin{abstract}
Background: Randomized trials show a mortality benefit to adjunctive corticosteroids for HIV-related Pneumocystis jiroveci pneumonia (HIV-PCP). Guidelines for non-HIV PCP (NH-PCP) recommend adjunctive corticosteroids based on expert opinion. We conducted a systematic review and meta-analysis characterizing adjunctive corticosteroids for NH-PCP.

Methods and findings: We searched MEDLINE from 1966 through 2015. Data on clinical outcomes from NH-PCP were extracted with a standardized instrument. Heterogeneity was assessed with the I2 index. Pooled odds ratios (OR) and 95\% confidence interval (95\% CI) were calculated using a fixed effects model. Our search yielded 5,044 abstracts, 277 articles were chosen for full review, and 6 manuscripts described outcomes in moderate to severe NH-PCP. Studies were limited by variable definitions, treatment selection bias, concomitant infections and small sample size. Individual studies reported shorter ICU stay and duration of mechanical ventilation of patients given adjunctive corticosteroids. There was no association between corticosteroids and survival in NH-PCP (OR 0.66; $95 \%$ CI: $0.38-1.15$, $\mathrm{p}=0.14)$.
\end{abstract}

Conclusions: The literature does not support an association between adjunctive corticosteroids and survival from NH-PCP but data are limited and findings should not be considered conclusive. Further research with improved methodology is needed to better understand the role of adjunctive corticosteroids for NH-PCP.

Abbreviations: PCP: Pneumocystis Jiroveci Pneumonia; H-PCP: HIV-associated PCP; NH-PCP: Non-HIV-associated PCP; CI: Confidence Interval; OR: Odds Ratio; PCR: Polymerase Chain Reaction; TMP-SMX: Trimethoprim-Sulfamethoxazole; ICU: Intensive Care Unit

\section{Introduction}

Research during the early AIDS epidemic fueled major advances in prevention and management of HIV-related Pneumocystis pneumonia (HIV-PCP) [1-4]. Specifically, the use of adjunctive corticosteroids for treatment of severe HIV-PCP ( $\mathrm{Pa} 02<70 \mathrm{mmHg}$ on room air) reduces mortality [5-7]. Non-HIV related Pneumocystis jirovecii pneumonia (NH-PCP) was previously considered a rare disease. However, with advances in immunomodulation and chemotherapeutics, NH-PCP is becoming more common. For example, NH-PCP complicates $1 \%$ of solid organ transplants and has a cumulative incidence of $0.1 \%$ per year for stem cell transplant recipients $[8,9]$.

There are no randomized trials to evaluate the effectiveness of adjunctive corticosteroids for NH-PCP. Despite lack of randomized trials, current guidelines recommend adjunctive corticosteroids for NH-PCP $[10,11]$. Retrospective studies with relatively small sample size have suggested either decreased mortality [12-15] or no impact
[16-19] with increasing the dosage of glucocorticoids as adjunctive treatment of moderate to severe NH-PCP. Older investigations, conducted when utilization of adjunctive glucocorticoid therapy was limited to patients with HIV-PCP, found higher mortality in NH-PCP compared to HIV-PCP [13,20-22]. More recent investigations have observed increased use of adjunctive glucocorticoids in NH-PCP, and similar mortality between HIV-PCP and NH-PCP [22-24]. With relatively limited available data to make guideline recommendations, the primary objective of this investigation was to systematically review the literature and perform a meta-analysis of available data relating to the use of use of adjunctive glucocorticoids in hospitalized patients with moderate to severe NH-PCP.

\section{Methods}

Search strategy and study selection: We performed a literature

Correspondence to: James A. Mckinnell, 1000 West Carson St, Box 466, Torrance CA 90501, USA; Tel: (310) 222-5639; Fax: (310) 861-8125; E-mail: Dr.Mckinnell@yahoo.com

Key words: pneumocystis, adjunctive therapy, corticosteroids, transplantation

Received: July 14, 2016; Accepted: August 19, 2016; Published: August 22, 2016 
Injean P (2016) A systematic review and meta-analysis of the data behind current recommendations for corticosteroids for non-HIV related PCP; Knowing when you are on shaky foundations

search of Medline from 1966 to July 2015 and of EMBASE from 1980 to July 2015 to find published manuscripts evaluating adjunctive glucocorticoid therapy for patients with NH-PCP. We limited studies to human subjects and searched for the following terms: (Pneumocysti ${ }^{\star}$ [text word] OR PCP[text word] OR "Pneumocystis Infections" [MESH] OR "Pneumocystis jirovecii" [MESH] OR "Pneumonia, Pneumocystis" [MESH] OR "Pneumocystis carinii" [MESH]) AND (treat ${ }^{*}$ [text word] OR adjunct* [text word] OR treatment ${ }^{\star}$ [text word] OR steroid ${ }^{\star}[$ text word] OR corticosteroid ${ }^{\star}$ [text word] OR glucocorticoid ${ }^{\star}$ [text word] OR "Glucocorticoids" [MESH] OR "Adrenal Cortex Hormones" [MESH] OR "Steroids" [MESH]) NOT ("animals"[MeSH] NOT "humans" [MeSH]) In addition, we examined the references of all identified articles to look for additional relevant articles. Non-English references were translated by an investigator (JM) or a native speaker whenever possible.

Abstracts from each reference from our electronic search were independently reviewed for relevance by two investigators (PI and JM) using a standardized instrument. Studies were selected for full review if they reported primary data from patients with NH-PCP treated with and without adjunctive corticosteroids. Studies that did not separate data on outcomes between patients treated with and without adjunctive corticosteroids were excluded. Reports of single case experiences were excluded on the basis of publication bias, e.g. exceptional circumstances surrounding the diagnosis, underlying conditions, or course of disease. There were no exclusions for patients with different types of immunosuppression or medical comorbidities. The intervention of interest was adjunctive corticosteroid therapy. The comparison groups were patients treated for $\mathrm{NH}-\mathrm{PCP}$ with and without adjunctive corticosteroids. The outcome of interest was mortality, as defined by the study investigators. The MOOSE criteria were used to conduct this investigation and the manuscript follows PRISMA criteria [25].

Data extraction: Each manuscript underwent independent, blinded, double-data extraction by two reviewers (JAM, PI) using a standardized instrument. Discrepancies in data extraction underwent arbitration by a third reviewer (AG) and consensus was obtained by verbal discussion. Data collected from each study included year of study, country, number of patients, method of PCP diagnosis, treatment of PCP, definition of adjunctive corticosteroid use, dose of steroids used, and mortality.

Additional data were collected about the patient cohorts when present, including ethnicity, age, comorbid conditions, malignancy, organ or stem cell transplant, immunological diseases. All-cause mortality and clinical cure rates, as defined by the individual studies, were the primary outcome measures used in this meta-analysis.

Data analysis and statistical methods: Data on NH-PCP outcomes were collected from all manuscripts. Odds ratios for mortality were calculated for each manuscript. Mantel-Haenszel statistical methods were used to calculate the pooled odds ratios, 95\% confidence intervals, and the associated p-values of each risk factor using a fixed-effects model. We analyzed heterogeneity in publication using the $\mathrm{I}^{2}$ measure of inconsistency and utilized DerSimonian and Laird random-effects model for $\mathrm{I}^{2}>50 \%$ or $\mathrm{P}<0.10$. We did not use additional weighting criteria for the analysis. To ensure that our results were not biased by the process of combining results from multiple investigations (i.e., Simpson's paradox), we present a forest-plot of data from each individual study $[26,27]$.

\section{Results}

Our search yielded 5,044 references possibly related to NH-PCP. After the abstracts of all references were reviewed, 4,767 abstracts were excluded because they were not related to PCP $(n=1,575)$, related to only H-PCP $(n=1,270)$, were basic science or animal models studies $(\mathrm{n}=893)$, related to prophylaxis only $(\mathrm{n}=377)$, a single case report $(n=374)$, or were only a literature review $(n=278)$. The full manuscript for 277 references were reviewed. From the 277 investigations with data on treatment of NH-PCP, 271 references were excluded from further analysis because they did not include outcome data $(n=81)$, did not have data on adjuvant steroid use $(n=65)$, untranslatable by the study team $(n=53)$, reported on HIV only $(n=28)$, reported single cases only $(n=20)$, outcomes were unclear $(n=15)$, reports of PCP colonization without evidence of infection $(n=7)$, or duplicated reports $(n=2)$.

In the final analysis, six retrospective cohort investigations of patients treated with adjunctive corticosteroids were included $[13,18]$ Among the six studies included in the analysis, there were 386 cases of NH-PCP. The six studies were conducted in the United States, France, Korea, and Japan. The patient population included solid organ transplantation $(n=111)$, collagen vascular disease $(n=25)$, rheumatoid arthritis $(n=21)$ hematological malignancies $(n=93)$, cancer $(n=11)$, nonhematologic malignancies $(n=29)$, interstitial lung disease $(n=9)$, connective tissue disease $(n=7)$, and other inflammatory diseases $(n=50)$. Diagnosis of NH-PCP was based on immunofluorescent staining, microscopic examination, or PCR of the patients' sputum, bronchoalveolar lavage fluid, or transbronchial biopsy.

The included investigations differed in their definition of adjuvant corticosteroid dose. (Table 1). The investigations defined adjuvant steroid use as; prednisone doses $>60 \mathrm{mg}$, pulse corticosteroids, high dose corticosteroids, adjunctive corticosteroids, prednisone doses $\geq 80$ $\mathrm{mg}$, and prednisone doses $>1 \mathrm{mg} / \mathrm{kg}$. Nearly all patients were initially treated with TMP-SMX prior to PCP infection.

Studies also differed in their definition of mortality. Three investigations used in hospital mortality rates, one investigation used 30day all-cause mortality, one used 30day and 90day all-cause mortality, and one used ICU mortality rates. A meta-analysis of the six studies showed treatment with adjuvant corticosteroids for $\mathrm{NH}$ PCP did not have an impact on survival (OR=0.76, 95\% CI 0.47-1.2;

Table 1. Papers Included in the Meta-analysis of the Impact of Adjunctive Corticosteroids on Survival from NH-PCP.

\begin{tabular}{|c|c|c|c|c|c|c|c|c|c|}
\hline Author & Year & Cohort & Country & Hospital & Adjuvant & Mortality & $\begin{array}{c}\text { No adjuvant } \\
\text { survival }\end{array}$ & $\begin{array}{l}\text { Adjuvant } \\
\text { survival }\end{array}$ & $\begin{array}{c}\text { Total number of } \\
\text { patients }\end{array}$ \\
\hline Pareja & 1998 & NH-PCP & USA & Teaching & Prednisone $>60 \mathrm{mg}$ & In-Hospital & $9 / 14(64 \%)$ & $9 / 16(56 \%)$ & 30 \\
\hline Aoki & 2009 & Hypoxic NH-PCP & Japan & Teaching & Pulse Corticosteroids & In-Hospital & $8 / 12(67 \%)$ & $6 / 13(46 \%)$ & 25 \\
\hline Komano & 2009 & Hypoxic NH-PCP & Japan & Multi Center & High Dose Corticosteroids & In-Hospital & $2 / 2(100 \%)$ & $19 / 19(100 \%)$ & 21 \\
\hline Matsumura & 2011 & NH- PCP & Japan & Tertiary & Adjuvant Corticosteroids & 30 days & $18 / 22(82 \%)$ & $44 / 60(73 \%)$ & 82 \\
\hline Moon & 2011 & Hypoxic NH-PCP & Korea & Teaching & Prednisone $\geq 80 \mathrm{mg}$ & $\begin{array}{l}30 \text { days } \\
90 \text { days }\end{array}$ & $19 / 29(66 \%)$ & $41 / 59(69 \%)$ & 88 \\
\hline Lemiale & 2013 & Hypoxic NH-PCP & France & Teaching & Prednisone $>1 \mathrm{mg} / \mathrm{kg}$ & ICU & $52 / 67(78 \%)$ & $51 / 72(71 \%)$ & 139 \\
\hline
\end{tabular}


Injean P (2016) A systematic review and meta-analysis of the data behind current recommendations for corticosteroids for non-HIV related PCP; Knowing when you are on shaky foundations

$\mathrm{p}=0.25)$. The most common co-infections described were CMV $(\mathrm{n}=23)$ and Aspergillus $(n=9)$. Patients were administered a wide range of immunosuppression at time of diagnosis. Reports of initial decline in respiratory function in patients not treated with corticosteroids was reported once [16].

\section{Discussion}

Non-HIV related Pneumocystis pneumonia is a growing problem in the United States, affecting the most vulnerable patient populations, including solid organ and stem cell transplant patients.

Our systematic review provides an important assessment of available literature on the role of adjunctive corticosteroids in the treatment of NH-PCP. We did not identify data from clinical trials. Ultimately, we found few papers that provided comparative data to assess the impact of adjunctive corticosteroids in NH-PCP. Based on our analysis of six manuscripts reporting on over 350 cases of $\mathrm{NH}$ $\mathrm{PCP}$, we found no association between adjunctive corticosteroids and survival in NH-PCP.

Our findings should not be considered conclusive. Despite the large number of cases, there were few investigations included in our analysis and all were retrospective in nature. Moreover, there was evidence for treatment selection bias in these investigations that was not adjusted in the original analysis and could not be adjusted for in our meta-analysis. We also caution that differences in definitions of mortality may further have introduced bias.

The effect of adjuvant corticosteroids on the duration of mechanical ventilation or ICU stay could not be assessed because only one out of six manuscripts included such intermediate outcomes data. Pareja et al found the ICU stay was shorter for patients treated with highdose steroids ( $\geq 60 \mathrm{mg} /$ day; $8.5 \pm 7$ days) compared to patients treated with low dose steroids or on a steroid taper ( $\leq 30 \mathrm{mg} /$ day; $15.8 \pm 8$ days) $(\mathrm{p}=0.025)$. The duration of mechanical ventilation was also significantly reduced in the increased high - dose steroid group (6.3 \pm 6 days) compared to the low dose/taper steroid group (18 \pm 21 days) $(p=0.047)$ [13]. While these data suggest potential benefit to the use of adjuvant corticosteroids, these results should be confirmed with additional studies.

There was relatively little safety data reported in these investigations. None of the manuscripts described incidence of hyperglycemia or other known side effects of corticosteroids, Moon et al, compared NHPCP patients treated with and without adjunctive corticosteroids and found no significant differences in the rates of concomitant bacterial infection, viral infections, or respiratory failure. Future investigations should carefully assess the safety of adjunctive corticosteroids on NH-PCP.

Overall, the impact of adjunctive corticosteroids on $\mathrm{NH}$ PCP remains unclear and requires further research. The currently published literature does not support a mortality benefit for adjunctive corticosteroids in NH-PCP, but the literature is limited to small, single center, retrospective cohort studies. Due to the inability to enroll an adequate number of subjects in a clinical trial, a standard prospective randomized clinical trial of NH-PCP is unlikely. Until such a trial is performed, we strongly believe that future research should make substantial changes in methodologies to provide more valuable insight. For example, a mortality endpoint, particularly a late mortality endpoint, may not be optimal in determining outcomes from NH-PCP as these patients have high risk for non-infection related deaths. Alternative outcome endpoints, including duration of ventilation, length of ICU stay, and the reporting on safety data would be valuable. Furthermore, Cox proportional hazards modeling, propensity score analysis, or instrumental variable analyses are needed to better understand outcomes from observations studies [28-30]. Until additional research using improved methodologies are published, clinicians should carefully consider the risk and benefits of adjunctive corticosteroids for NH-PCP $[10,11]$.

\section{Acknowledgements}

PI received support from the Western University of Health Sciences Research Committee. JM received support from the NIH/ NCRR/NCATS UCLA CTSI Grant Number KL2TR000122 and the Perkins Foundation. ALG received support from the NIH/NHLBI Grant Number K23HL102220.

\section{Disclosures}

None

\section{Authorship}

PI, ALG, JAM: conception, hypothesis, data review and generation, manuscript development and revision SJE: Statistical analysis, manuscript revision HW, IM: data review, manuscript revision.

\section{References}

1. Gagnon S, Boota AM, Fischl MA, Baier H, Kirksey OW, et al. (1990) Corticosteroids as adjunctive therapy for severe Pneumocystis carinii pneumonia in the acquired immunodeficiency syndrome. A double-blind, placebo-controlled trial. $N$ Engl J Med 323: 1444-1450. [Crossref]

2. Bozzette SA, Sattler FR, Chiu J, Wu AW, Gluckstein D, et al. (1990) A controlled trial of early adjunctive treatment with corticosteroids for Pneumocystis carinii pneumonia in the acquired immunodeficiency syndrome. California Collaborative Treatment Group. N Engl J Med 323: 1451-1457. [Crossref]

3. Montaner JS, Lawson LM, Levitt N, Belzberg A, Schechter MT, et al. (1990) Corticosteroids prevent early deterioration in patients with moderately severe Pneumocystis carinii pneumonia and the acquired immunodeficiency syndrome (AIDS). Ann Intern Med 113: 14-20. [Crossref]

4. Kaplan JE, Hanson D, Dworkin MS, Frederick T, Bertolli J, et al. (2000) Epidemiology of human immunodeficiency virus-associated opportunistic infections in the United States in the era of highly active antiretroviral therapy. Clin Infect Dis 30 Suppl 1: S5-14. [Crossref]

5. Kaplan JE, Masur H, Holmes KK. (2002) Guidelines for preventing opportunistic infections among HIV-infected persons--2002. Recommendations of the U.S. Public Health Service and the Infectious Diseases Society of America. MMWR Recomm Rep 51(RR-8): 1-52. [Crossref]

6. Huang L, Morris A, Limper AH, Beck JM; ATS Pneumocystis Workshop Participants (2006) An Official ATS Workshop Summary: Recent advances and future directions in pneumocystis pneumonia (PCP). Proc Am Thorac Soc 3: 655-664. [Crossref]

7. Briel M, Bucher HC, Boscacci R, Furrer H. (2006) Adjunctive corticosteroids for Pneumocystis jiroveci pneumonia in patients with HIV-infection. Cochrane Database Syst Rev 3: CD006150. [Crossref]

8. Kontoyiannis DP, Marr KA, Park BJ, Alexander BD, Anaissie EJ, et al. (2010) Prospective Surveillance for Invasive Fungal Infections in Hematopoietic Stem Cell Transplant Recipients, 2001-2006: Overview of the Transplant-Associated Infection Surveillance Network (TRANSNET) Database. Clin Infect Dis 50: 1091-1100. [Crossref]

9. Pappas PG, Alexander BD, Andes DR, Hadley S, Kauffman CA, et al. (2015) Invasive Fungal Infections among Organ Transplant Recipients: Results of the TransplantAssociated Infection Surveillance Network (TRANSNET). Clin Infect Dis 50: 11011011. [Crossref]

10. Martin SI, Fishman JA, Practice ASTIDCo. (2013) Pneumocystis pneumonia in solid organ transplantation. Am J Transplant 13 Suppl 4: 272-279. [Crossref]

11. McKinnell JA, Cannella AP, Injean P, Gregson A. (2014) Adjunctive glucocorticoid therapy for non-HIV-related pneumocystis carinii pneumonia (NH-PCP). Am J Transplant 14: 982-983. 
Injean P (2016) A systematic review and meta-analysis of the data behind current recommendations for corticosteroids for non-HIV related PCP; Knowing when you are on shaky foundations

12. Burke BA, Good RA (1973) Pneumocystis carinii infection. Medicine (Baltimore) 52: 23-51. [Crossref]

13. Pareja JG1, Garland R, Koziel H (1998) Use of adjunctive corticosteroids in severe adult non-HIV Pneumocystis carinii pneumonia. Chest 113: 1215-1224. [Crossref]

14. Matsumura Y, Shindo Y, Iinuma Y, Yamamoto M, Shirano M, et al. (2011) Clinical characteristics of Pneumocystis pneumonia in non-HIV patients and prognostic factors including microbiological genotypes. BMC Infect Dis 11: 76. [Crossref]

15. Aoki Y, Iwamoto M, Kamata Y, Nagashima T, Yoshio T, et al. (2009) Prognostic indicators related to death in patients with Pneumocystis pneumonia associated with collagen vascular diseases. Rheumatol Int 29: 1327-1330. [Crossref]

16. Moon SM, Kim T, Sung H, Kim MN, Kim SH, et al. (2011) Outcomes of moderate-tosevere Pneumocystis pneumonia treated with adjunctive steroid in non-HIV-infected patients. Antimicrob Agents Chemother 55: 4613-4618. [Crossref]

17. Komano Y, Harigai M, Koike R, Sugiyama H, Ogawa J, et al. (2009) Pneumocystis jiroveci pneumonia in patients with rheumatoid arthritis treated with infliximab: a retrospective review and case-control study of 21 patients. Arthritis Rheum 61: 305312. [Crossref]

18. Lemiale V, Debrumetz A, Delannoy A, Alberti C, Azoulay E. (2013) Adjunctive steroid in HIV-negative patients with severe Pneumocystis pneumonia. Respir Res 14: 87. [Crossref]

19. Zahar JR1, Robin M, Azoulay E, Fieux F, Nitenberg G, et al. (2002) Pneumocystis carinii pneumonia in critically ill patients with malignancy: a descriptive study. Clin Infect Dis 35: 929-934. [Crossref]

20. Limper AH, Offord KP, Smith TF, Martin WJ, 2nd. (1989) Pneumocystis carinii pneumonia. Differences in lung parasite number and inflammation in patients with and without AIDS. T Am Rev Respir Dis 140: 1204-1209. [Crossref]

21. Kovacs JA, Hiemenz JW, Macher AM, Stover D, Murray HW, et al. (1984) Pneumocystis carinii pneumonia: a comparison between patients with the acquired immunodeficiency syndrome and patients with other immunodeficiencies. Ann Intern Med 100: 663-671. [Crossref]

22. Mansharamani NG, Garland R, Delaney D, Koziel H. (2000) Management and outcome patterns for adult Pneumocystis carinii pneumonia, 1985 to 1995: comparison of HIVassociated cases to other immunocompromised states. Chest 118: 704-711. [Crossref]

23. Nuesch R, Bellini C, Zimmerli W. (1999) Pneumocystis carinii pneumonia in human immunodeficiency virus (HIV)-positive and HIV-negative immunocompromised patients. Clin Infect Dis 29: 1519-1523. [Crossref]

24. McKinnell JA, Cannella AP, Kunz DF, Hook EW, 3rd, Moser SA, et al. (2012) Pneumocystis pneumonia in hospitalized patients: a detailed examination of symptoms, management, and outcomes in human immunodeficiency virus (HIV)-infected and HIV-uninfected persons. Transpl Infect Dis 14: 510-518. [Crossref]

25. Stroup DF, Berlin JA, Morton SC, Olkin I, Williamson GD, et al. (2000) Meta-analysis of observational studies in epidemiology: a proposal for reporting. Meta-analysis Of Observational Studies in Epidemiology (MOOSE) group. Jama 283: 2008-2012. [Crossref]

26. Bickel PJ, Hammel EA, O'connell JW (1975) Sex bias in graduate admissions: data from berkeley. Science 187: 398-404. [Crossref]

27. Pearl J. (2000) Causality: models, reasoning, and inference. Cambridge, U.K ; New York: Cambridge University Press. xvi, 384 p. p.

28. Berger ML, Mamdani M, Atkins D, Johnson ML. (2009) Good research practices for comparative effectiveness research: defining, reporting and interpreting nonrandomized studies of treatment effects using secondary data sources: the ISPOR Good Research Practices for Retrospective Database Analysis Task Force Report--Part I. Value Health 12: 1044-1052. [Crossref]

29. Johnson ML, Crown W, Martin BC, Dormuth CR, Siebert U. (2009) Good research practices for comparative effectiveness research: analytic methods to improve causal inference from nonrandomized studies of treatment effects using secondary data sources: the ISPOR Good Research Practices for Retrospective Database Analysis Task Force Report--Part III. Value Health 12: 1062-1073. [Crossref]

30. Cox E, Martin BC, Van Staa T, Garbe E, Siebert U, Johnson ML. (2009) Good research practices for comparative effectiveness research: approaches to mitigate bias and confounding in the design of nonrandomized studies of treatment effects using secondary data sources: the International Society for Pharmacoeconomics and Outcomes Research Good Research Practices for Retrospective Database Analysis Task Force Report--Part II. Value Health 12: 1053-1061. [Crossref]

Copyright: (C2016 Injean P. This is an open-access article distributed under the terms of the Creative Commons Attribution License, which permits unrestricted use, distribution, and reproduction in any medium, provided the original author and source are credited. 